\title{
Time-Domain Optical Coherence Tomography System for Determining The Extinction Coefficient and Group Refractive Index of Gelatin-based Skin Phantoms
}

\author{
Maria Cecilia Galvez ${ }^{1,2 *}$, Edgar Vallar ${ }^{1,2}$, Tatsuo Shiina ${ }^{3}$, Ernest Macalalad ${ }^{4}$, Paulito Mandia ${ }^{1,2}$ \\ ${ }^{1}$ Environment and Remote Sensing Research Laboratory, Physics Department, De La Salle University, Manila, 2401, Philippines

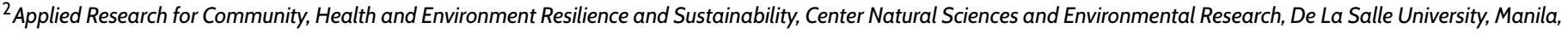 \\ 2401, Philippines \\ ${ }^{3}$ Graduate School of Science and Engineering, Chiba University, Chiba, 271-8510, Japan \\ ${ }^{4}$ Department of Physics, Mapúa University, Manila, 1002, Philippines \\ *Corresponding author: maria.cecilia.galvez@dlsu.edu.ph
}

\begin{abstract}
Optical Coherence Tomography (OCT) is a non-invasive, non-destructive optical imaging technique that uses a low coherence interferometer to obtain real-time cross-sectional images of samples. OCT is notably used in biomedical applications including ophthalmology and dermatology. Aside from generating cross-sectional images, axial scans can also provide additional information about its optical properties such as extinction coefficient and refractive index. This study determines the extinction coefficients and group refractive indices of gelatin-based skin phantoms using a portable time-domain (TD) - OCT system. The gelatin-based skin phantoms were fabricated with varying concentrations of titanium dioxide $\left(\mathrm{TiO}_{2}\right)$, while keeping the amount of both gelatin and water constant. By changing the proportion of the gelatin powder and $\mathrm{TiO}_{2}$, skin phantoms can then be fabricated to mimic various skin conditions, both pathologic and non-pathologic. Results of the study found a positive correlation of extinction coefficient and refractive index with $\mathrm{TiO}_{2}$ concentration. Thus, increasing $\mathrm{TiO}_{2}$ concentration also increases both extinction coefficient and group refractive index. The median extinction coefficient values of the phantoms ranged from $4.29 \mathrm{~mm}^{-1}$ to $8.48 \mathrm{~mm}^{-1}$. Literature showed that the epidermis can have extinction coefficients of $1.64-7.3 \mathrm{~mm}^{-1}$. For refractive indices of the fabricated phantoms, values ranged from 1.32 to 1.48 , while studies on human participants showed that human skin has refractive index values of 1.34-1.56. Based on these properties, it is feasible to fabricate phantoms simulating the optical properties of human skin.
\end{abstract}

Keywords

Optical Coherence Tomography, Extinction Coefficient, Refractive Index, Gelatin, Skin Phantom

Received: 27 June 2021, Accepted: 18 September 2021

https://doi.org/10.26554/sti.2021.6.4.319-327

\section{INTRODUCTION}

Optical coherence tomography is an imaging modality that uses the interference of low-coherence light to generate a crosssectional image of the sample (Fujimoto and Drexler, 2015). It was first demonstrated on the retina (Fujimoto et al., 1991). Until now, the most popular application of OCT is in biomedical imaging, particularly in ophthalmology. Other organs are also under study such as the skin (Schneider et al., 2019) and gastrointestinal tract (Pant et al., 2017). Today, OCT has found widespread use in various fields such as agriculture ( $\mathrm{Ra}-$ teria et al., 2019) and forensics (Marques et al., 2021). The reason for its popularity in medicine is that it can produce highresolution histological images of the tissue without the need for biopsy or dissection, hence the term "optical biopsy". Similarly, this is a valuable tool in industry since OCT can characterize materials without destroying the sample (Fujimoto and Drexler, 2015; Izatt et al., 2015).

The first type of OCT constructed was the time-domain OCT (TD-OCT) wherein the signal is a function of time. TD-OCT uses a movable reference mirror. A newer type is the frequency-domain OCT (FD-OCT) wherein the signal obtained is a function of frequency. This type has a fixed reference mirror. The absence of a movable mirror gives FDOCT an advantage in terms of data acquisition time. That is why FD-OCT is more popular nowadays than TD-OCT. But what makes TD-OCT still viable until today is that it has a simple design and its much cheaper components (Liu, 2014).

The skin is the outermost organ of the human body. It is exposed daily to a harsh environment not limited to extremes of temperature, radiation, corrosive chemicals, mechanical trauma, and pathogens. As a result, numerous skin conditions 
develop, as manifested by discoloration, abnormal growth, etc., among other changes.

When doing studies of OCT on biological tissues such as skin, a particular tissue type may not always be available as in the case of rare diseases. Even among healthy individuals, skin optical properties may also vary due to anatomic location, age, sex, occupation, and many other factors (Gambichler et al., 2006; James et al., 2020). This can be a problem when designing OCT parts since replicability of the experiments could be limited. That is why there are situations in which samples with consistent properties should be available even with several repetitions. This can be addressed by fabricating tissue models. In the biomedical field, these models that mimic certain properties (optical, mechanical, acoustic, etc.) of tissues are called tissue phantoms (Cook et al., 2011). The tissue properties of these phantoms can be easily adjusted by varying the proportions of the ingredients. Soon, when OCT could be used as an aid to invasive medical procedures, pain and discomfort can be avoided by practicing on inanimate models first before attempting on actual patients. Aside from practicality, the use of tissue phantoms can be an ethical option as well (Kim, 2016).

We undertook this research to determine the extinction coefficients and refractive indices of fabricated gelatin-based skin phantoms using a TD-OCT constructed in our lab. This will enable us to fabricate tissue phantoms and adjust their optical properties, i.e., extinction coefficient and refractive index, that can then be used to simulate different skin conditions, both physiologic and pathologic. Several OCT studies with tissue phantoms have already been undertaken, such as those of Vermeer et al. (2014) who used silicone as base material, while Adili and Shiina (2018) used model paint. Both papers stated that increasing $\mathrm{TiO}_{2}$ concentration also increased the extinction coefficient of their tissue phantoms. It was demonstrated that solute concentrations do affect refractive index values, such as the work of Forouzeshfard et al. (2021) using aqueous solutions of sodium chloride and ethanol in their development of a metamaterial sensor. Conversely, solute concentration can be determined by measuring the refractive index of a solution, such as glucose measurement through interferometry by Yeh (2008). Gelatin- $\mathrm{TiO}_{2}$ phantoms are commonly used outside of OCT as well. Akarçay et al. (2012) determined that $\mathrm{TiO}_{2}$ concentration increases the extinction coefficient through computer simulation and spectrometry at $780 \mathrm{~nm}$ wavelength.

\section{EXPERIMENTAL SECTION}

\subsection{Working Principles of TD-OCT}

The working principle of TD-OCT is based on Michelson Interferometer. The schematic diagram of a TD-OCT system is shown in Figure 1. Light is emitted by a broadband light source, such as a superluminescent diode (SLD) connected to a fiber coupler. Light is split in two by a beam splitter usually in a 50:50 ratio. One portion of light travels along the sample arm, exits through the probe, and penetrates the sample. The probe consists of a collimator and a focusing lens. As light penetrates the sample, it hits reflective and scattering

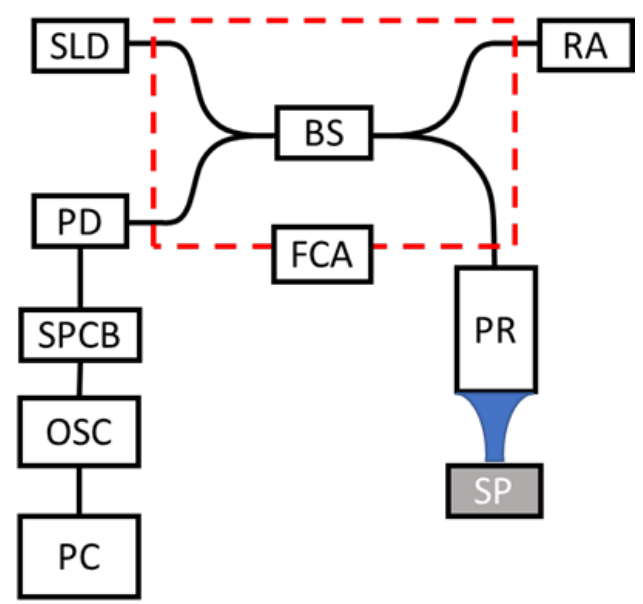

Figure 1. The Schematic Diagram of the TD-OCT System. SLD, Superluminescent Diode; PD, Photodiode; SPCB, Signal Processing Circuit Board; OSC, Oscilloscope; PC, Personal Computer; BS, Beam Splitter; RA, Reference Arm; PR, Probe; SP, Sample; FCA, Fiber Coupler Assembly (Enclosed in Red Box)

boundaries such as particles. The portion of light scattered back into the probe, travels through the fiber optics, and then collected by a photodiode detector (PD). The other portion of light from the beam splitter travels along the reference arm, exits through a collimator, and hits the reference mirror. Instead of having translational motion, as traditionally used in Michelson interferometers, the reference mirror is modified into a rotating retroreflector as described by Shiina et al. (2003). The advantage of a rotating mechanism over a translational one is a longer scanning range, which can be easily adjusted by changing the rotational radius of the retroreflector. The optical path difference is almost linear at angles less than \pm 20 degrees. The repeatability of scanning is improved when using a rotating reflector. Additionally, the scanning speed can also be adjusted (Shiina et al., 2003). Moreover, such flexibility of the design can be implemented while maintaining the compactness and portability of the system. As in the case of the sample arm, the reflected light from the reference mirror, reenters the collimator, travels through the fiber optics and then collected by the PD. Interference can occur whenever the path length difference between the backscattered light from the sample arm and the reflected light from reference arm is close to the value of the coherence length, which is a quantity that depends on the bandwidth of the light source. The interference signal is converted into an electrical signal in the receiver circuit, and the envelope of the interferogram is displayed on the oscilloscope. The oscilloscope generates a signal in the time domain, which is an array of time and light intensities. This signal carries the depth information of the sample. The signal carrying the depth information of a single point is called an axial scan (A-scan). When the sample is moved along a straight line perpendicular to the probe axis, adjacent points of the sample can then be 
scanned. By combining the A-scans of these adjacent points, a B-scan can be produced, which is simply the cross-sectional image of the sample. In FD-OCT, the signal is first inverse Fourier transformed to convert it into time-domain (Izatt et al., 2015).

While OCT is mainly known for its B-scan images, the A-scan can also reveal important information about the optical properties of the sample, such as the extinction coefficient and the refractive index. To better understand the extinction coefficient, let us first look at Equation (1) which is a modified Beer-Lambert's law (Chang and Bowden, 2019):

$$
I=I_{0} e^{-2 \mu z}
$$

where $I$ is the intensity of light upon reaching the PD, $I_{0}$ is the incident light from the probe, $\mu$ is the extinction coefficient, and $\mathrm{z}$ is the depth traveled by light along the $\mathrm{z}$-axis. The negative exponent shows that the intensity of light decreases as it travels through a medium. The number " 2 " in the exponent means the round trip travel of light from the probe to the sample, and back to the probe. The amount of decrease or attenuation not only depends on the depth but also on the optical property of the tissue which is the extinction (or attenuation) coefficient. A large value of $\mu$ means that light is strongly attenuated in that medium and thus the depth of penetration is severely limited.

By cross-multiplying $I_{0}$ and then taking the natural logarithm of both sides gives the logarithmic form of Beer-Lambert's law (Adili and Shiina, 2018):

$$
\ln \left(\frac{I}{I_{0}}\right)=-2 \mu z
$$

This is the linearized form of the Beer-Lambert equation. By plotting $\ln \left(I / I_{0}\right)$ onto the $\mathrm{y}$-axis and depth $\mathrm{z}$ onto the $\mathrm{x}$-axis, the slope of the curve-fitted line contains the extinction coefficient, similar to the work of Faber et al. (2004). The extinction coefficient $\mu$ referred here is the total extinction coefficient defined as $\mu=\mu_{s}+\mu_{a}$, where $\mu_{s}$ is called the scattering coefficient and $\mu_{a}$ is the absorption coefficient. For most biological tissues, which are predominantly composed of water, the scattering coefficient is 10-100 times greater than the absorption coefficient (Cheong et al., 1990; Ugryumova et al., 2004; Tuchin et al., 2011). Hence for practical purposes $\mu \approx \mu_{s}$.

On the other hand, the absolute refractive index $\mathrm{n}$ is the ratio between the speed of light in free space and the speed of light in matter (in most literature, absolute refractive index is simply referred to as refractive index). Absolute refractive index is used for systems with monochromatic light where dispersion effects due to varying wavelengths can be ignored. But for a system such as OCT which uses a broadband light source, a more accurate quantity to use is the group refractive index, $n_{g}$, defined as

$$
n_{g}=c / v_{g}
$$

where $c$ is the magnitude of the velocity of light in free space, and $v_{g}$ is the group velocity, or the rate at which the modulation envelope of a group of waves advances (Hecht, 2017).

These optical properties are of particular interest to us since biological tissues also have their own unique sets of optical properties. Furthermore, tissue optical properties can be altered with the presence of disease. For example, Yang et al have shown that tissue samples with oral squamous cell carcinoma, a type of cancerous lesion, has a lower extinction coefficient compared to that of normal tissue (Yang et al., 2020). The increased cell density in cancerous tissue meanwhile increases the overall refractive index of the lesion (Khan et al., 2021). Thus, by studying the cross-sectional image together with the extinction coefficients and the refractive indices, we can improve the accuracy of disease diagnosis.

\subsection{TD-OCT System}

The portable TD-OCT system in this study used a $1310 \mathrm{~nm}$ SLD manufactured by Anritsu Co. Ltd. with a spectral width of $106 \mathrm{~nm}$. These properties of the SLD determines the axial resolution of the OCT system, which in this case is $7 \mu \mathrm{m}$ in air. The lateral resolution which depends on the numerical aperture of the sample probe, is $3 \mu \mathrm{m}$. As described previously, the optical path scanning mechanism of our OCT system is a rotating mirror with a scanning diameter of $10 \mathrm{~mm}$ and a scanning rate of $25 \mathrm{scan} / \mathrm{s}$. A more detailed discussion on this optical path scanning mechanism was discussed by Shiina et al. (2003). The specifications of the TD-OCT system are summarized on Table 1.

Table 1. Specifications of The TD-OCT System

\begin{tabular}{cc}
\hline Specification & Value \\
\hline Center wavelength & $1310 \mathrm{~nm}$ \\
Spectral width & $106 \mathrm{~nm}$ \\
Axial resolution & $7 \mu \mathrm{m}$ \\
Lateral resolution, spot size & $6 \mu \mathrm{m}$ \\
Numerical aperture & 0.14 \\
Scanning rate & $25 \mathrm{scans} / \mathrm{s}$ \\
Scanning depth in air & $12-14 \mathrm{~mm}$ \\
\hline
\end{tabular}

\subsection{Fabrication of Gelatin- $\mathrm{TiO}_{2}$-based Phantoms}

In preparing for the experiment, phantoms were fabricated first using $50 \mathrm{ml}$ of water, $3 \mathrm{~g}$ of gelatin as the base material, and titanium dioxide as the scattering agent with varying percent concentrations by mass, namely, $0.02 \%, 0.03 \%, 0.09 \%, 0.13 \%$, and $0.17 \%$. The $\mathrm{TiO}_{2}$ particle size of the $\mathrm{TiO}_{2}$ powder was 0.3 $\mu \mathrm{m} \pm 0.05 \mu \mathrm{m}$.

On a beaker with $20 \mathrm{ml}$ distilled water at room temperature, gelatin powder was poured, followed by $\mathrm{TiO}_{2}$ powder. Gelatin was poured first to since the researcher observed that when $\mathrm{TiO}_{2}$ adheres to the bottom of the beaker, it was difficult to scrape with a stirring rod. Also, it is important to break up the 


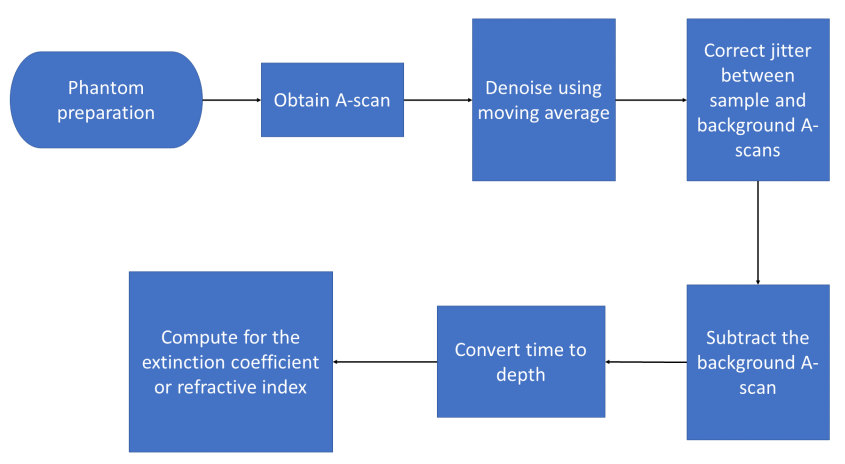

Figure 2. Workflow for Data Acquisition and Processing

clumps before pouring the $\mathrm{TiO}_{2}$ powder. The mixture was mixed lightly, and gelatin was left to soak for around 5 minutes.

Next, $30 \mathrm{ml}$ of distilled water preheated to $60-65^{\circ} \mathrm{C}$ was poured onto the soaked solution and stirred to produce a homogeneous mixture. The amount of stirring was limited to minimize the formation of air bubbles. After this, $7 \mathrm{ml}$ of the solution was poured onto its corresponding petri dish using a syringe. Another $0.2-0.25 \mathrm{ml}$ was injected into its corresponding refractive index sample holder. In both samples, air bubbles were removed using the syringe needle. For each $\mathrm{TiO}_{2}$ concentration, two solutions were mixed and placed on two separate petri dishes and two separate refractive index sample holders.

It is important to note that while pouring hot water into the graduated cylinder, the water cooled down by as much as $15^{\circ} \mathrm{C}$. Further cooling occurs when water is poured into the soaked gelatin. Hence before measurement, an initial amount of water was poured into the cylinder first and discarded before pouring the actual amount of water to be mixed with gelatin. This is done so that by the time the $30 \mathrm{ml}$ warm water is mixed with the soaked gelatin, the temperature would be around 40-50 ${ }^{\circ} \mathrm{C}$, which was about right to melt gelatin and form gel, but not high enough to degrade gel strength (GMIA, 2019).

To minimize the settling of particles and maintain the homogeneity of the phantom, the warm samples were cooled in an ice box at $10{ }^{\circ} \mathrm{C}$ to accelerate gelation. When ready, the samples were brought out and left to gradually attain room temperature before data gathering. The petri dishes were covered to prevent the escape of moisture from the samples.

\subsection{Methods}

\subsubsection{Data Acquisition and Processing}

Prior to data gathering, the phantoms were first brought out of the ice box so that the phantoms will attain a room temperature of around $210^{\circ} \mathrm{C}$. To acquire data, the probe was oriented perpendicular to the sample surface. Once an A-scan is generated, the data was saved as a CSV file which was further processed as outlined in Figure 2.

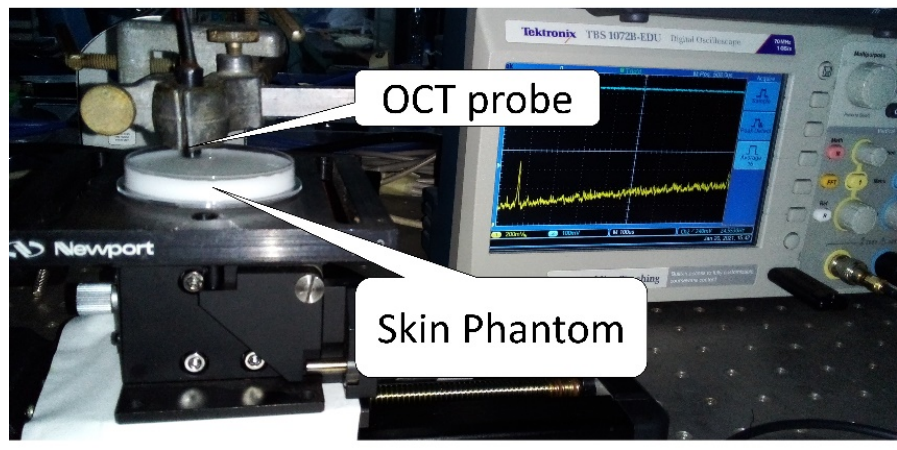

Figure 3. The Setup for Extinction Coefficient Determination

\subsubsection{Extinction Coefficient Determination}

The petri dish was placed under the probe, as seen in Figure 3 , where the probe was oriented perpendicular to the sample's surface. For each of the five $\mathrm{TiO}_{2}$ concentration, two skin phantoms were prepared, for a total of ten phantoms. Also, for each concentration, a total 11-13 of points along the surface were selected, with each point being an average of $128 \mathrm{~A}$-scans.

When positioning the probe and the sample, the working distance was adjusted so that the peak corresponding to the boundary between air and the sample would always have the highest intensity. This places the probe's focal length near the surface of the sample. Since the samples were homogenous, the waveforms should have a general downward trend. This is exemplified by Figure 4(a), which is one of the A-scan signals of the $0.17 \%$ phantom. For convenience, the signal was shifted along the $\mathrm{x}$-axis such that the start of the surface peak is designated as the $0 \mathrm{~mm}$ depth. Oftentimes the surface signal can be saturated hence the clipped (flat) peak. This happens when the voltage is beyond the viewing range of the oscilloscope. Thus, in taking the extinction coefficient, the surface peak is not included in the curve-fitting. In the oscilloscope, the $\mathrm{x}$-axis is measured as time in milliseconds. Time is then converted to depth by multiplying with a conversion factor dependent on the size and insertion angle of the rotating retroreflector (Shiina et al., 2003).

Once a proper table of signal intensity versus depth was obtained, the natural logarithm of the signal was computed, shown in Figure 4(b), after which a segment of the plot was selected, beginning from the peak next to the surface until the curve reaches the baseline. Curve-fitting was performed on the selected segment using the 'polyfit' function of Matlab ${ }^{\mathrm{TM}}$ (the same results can be obtained using the 'trendline' feature of MS-Excel ${ }^{\mathrm{TM}}$ ). Figure 4(b) shows the natural logarithm of the same A-scan as Figure 4(a), with the orange line being the segment that was curve-fitted. The black dashed line is the curve-fit, whose slope divided by -2 is the extinction coefficient.

To verify the relationship between the extinction coefficient and $\mathrm{TiO}_{2}$ concentration, transmittances of the phantoms were obtained using an optical power meter. Transmittance is simply 


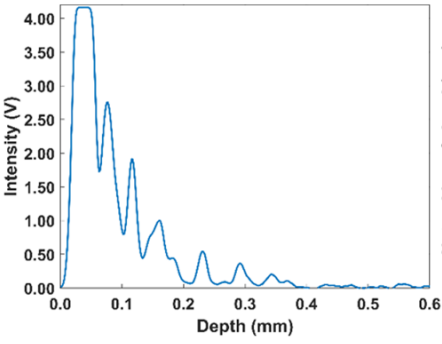

(a)

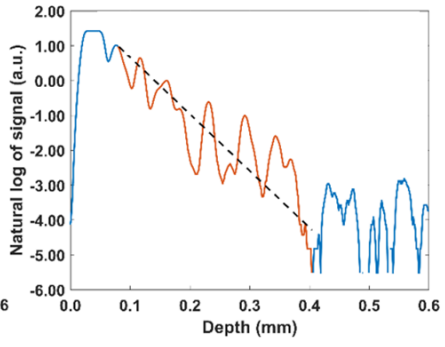

b)
Figure 4. An A-Scan of the $0.17 \%$ Skin Phantom. (a) The Appearance of An A-Scan; (b) The Same Signal after Taking The Natural Logarithm of Intensity.

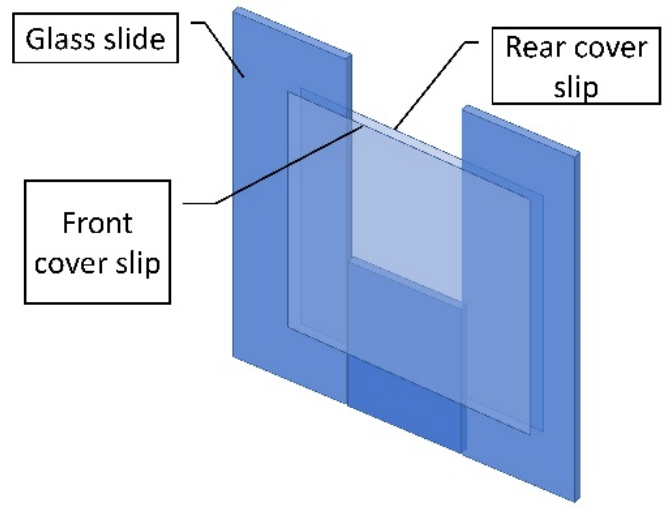

Figure 5. The Sample Holder used to Contain The Skin Phantom for Refractive Index Computation

the ratio of the incident power from the light source $\mathrm{P}_{0}$ and the resulting power $\mathrm{P}$. Incident power $\mathrm{P}_{0}$ was obtained by measuring the light passing through an empty petri dish. Resulting power $\mathrm{P}$ was obtained by measuring the light passing through the same petri dish containing the phantom. Going back to Beer-Lambert's law, a higher extinction coefficient should give a lower transmittance. For the transmittance, the same samples as those in extinction coefficient determination were used. Measurement time was 10 seconds with a sampling resolution of 10 milliseconds. For each phantom, three repetitions were done.

\subsubsection{Group Refractive Index Determination}

A sample holder was constructed out of two glass coverslips separated by cut glass slides held together by waterproof glue as shown in Figure 5.

The OCT probe and the sample holder were oriented like in Figure 6. The distance was adjusted so that the peaks corresponding to the front and rear surfaces of the sample were visible. Especially for higher $\mathrm{TiO}_{2}$ concentrations, the probe was positioned closer to the sample holder. Signal intensities do not matter for this purpose since only the optical path lengths are required for the calculation. First, A-scans of the empty sample holders were taken. Each sample holder was then filled

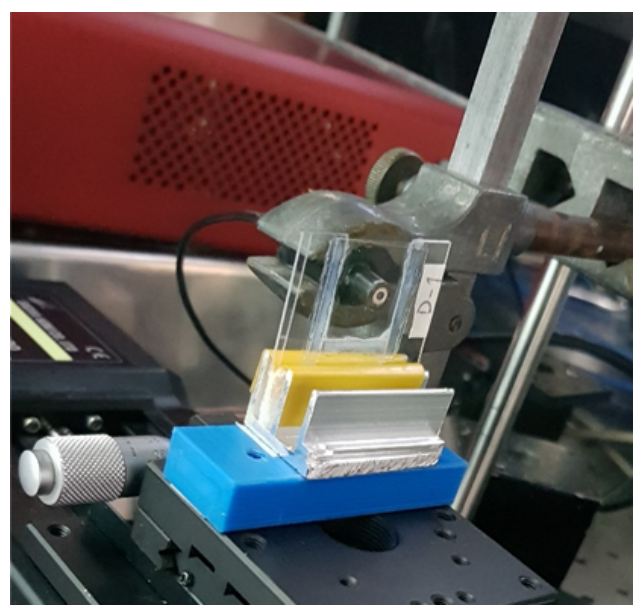

Figure 6. The Setup for Refractive Index Determination

with liquid phantom and left until each phantom will form gels. Similar as the extinction coefficient determination, for each of the five $\mathrm{TiO}_{2}$ concentration, two samples were prepared, for a total of ten samples. For each concentration, 5-10 points were selected, with each point being an average of $128 \mathrm{~A}$-scans. To compute for the refractive index, the following equation was used (Ugryumova et al., 2004):

$$
\Delta=n_{g} d
$$

where $\Delta$ is the optical thickness, $n_{g}$ is the group refractive index of the sample and $d$ is the thickness of the cavity of the sample holder. Therefore, by knowing the optical and physical thickness, the group refractive index can be calculated (Maruyama et al., 2002). Prior to phantom preparation, A-scans of the empty sample holders were taken. The thickness of the empty sample holder served as the physical thickness since the refractive index of air is almost equal to that of free space. Next, the sample holder was injected with water, and the optical thickness was taken. With this, the group refractive index of water can be calculated using Equation (4). The sample holder was then emptied, air-dried, and the phantom was injected. The group refractive indices of the skin phantoms were computed in a similar manner.

\section{RESULTS AND DISCUSSION}

\subsection{Extinction Coefficient}

Table 2 shows the mean, median, and standard deviation of the extinction coefficients per concentration of $\mathrm{TiO}_{2}$. Figure 7 is a whisker plot showing the relationship between extinction coefficient and titanium dioxide concentration. Except for the $0.02 \%$ mixture, there is an overall upward trend in the extinction coefficient as the concentration of $\mathrm{TiO}_{2}$ increases. The higher the extinction coefficient, the larger is the amount of light attenuated as it travels deeper into the phantom.

Based on figure 7 , the obtained extinction coefficient at $0.02 \%$ phantom has a wide spread of values, producing data 
Table 2. Extinction Coefficient for each $\mathrm{TiO}_{2}$ Concentration

\begin{tabular}{cccc}
\hline $\mathrm{TiO}_{2}$ Concentration & \multicolumn{3}{c}{ Extinction Coefficients $\left(\mathrm{mm}^{-1}\right)$} \\
(\% by mass) & Average & Median & Standard Deviation \\
\hline 0.02 & 7.05 & 8.48 & 4.33 \\
0.06 & 4.4 & 4.88 & 1.12 \\
0.09 & 5.08 & 4.29 & 1.87 \\
0.13 & 6.72 & 6.12 & 1.92 \\
0.17 & 7.01 & 7.23 & 1.14 \\
\hline
\end{tabular}

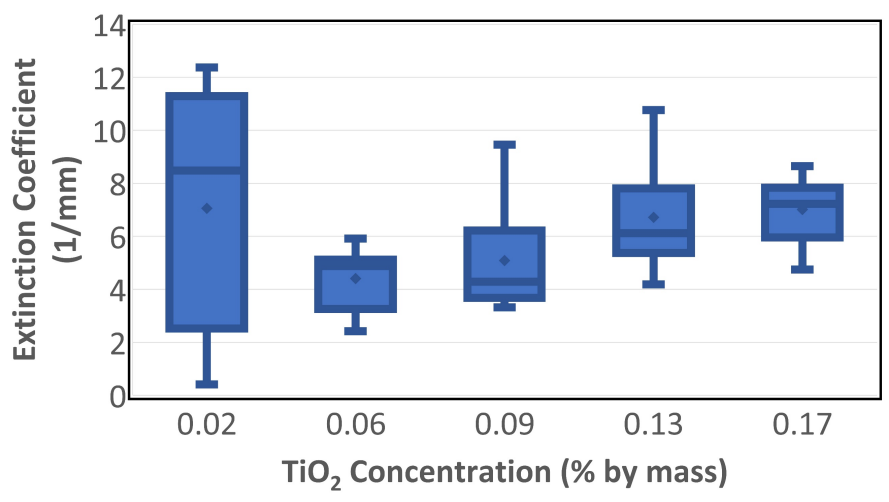

Figure 7. The Plot showing the Relationship between Extinction Coefficient and $\mathrm{TiO}_{2}$ Concentration

with higher standard deviations. Larger errors in the lowconcentration section are partly due to the fact that having less particles at low-concentrations leads to low values and a slow variation of backscatter intensity and it causes significant scattering intensity fluctuations (Huang et al., 2019; Yang et al., 2008). Conversely, at higher concentration of $\mathrm{TiO}_{2}$, there would be more scattering particles and a higher chance to detect points with high scattering intensity, hence, obtaining a more consistent signal intensities along the scanning region (Yang et al., 2008).

An earlier study was done by Vermeer et al. (2014) wherein they used $\mathrm{TiO}_{2}$ as the scattering agent and silicone as the base material. Their results also showed an increase in extinction coefficient with $\mathrm{TiO}_{2}$ concentration. Several OCT studies have been done to determine the extinction coefficient of the human skin. Using a $1310 \mathrm{~nm}$ light source, Adili and Shiina (2018) found that the extinction coefficient of the forearm was $7 \mathrm{~mm}^{-1}$ and the upper arm $7.3 \mathrm{~mm}^{-1}$. Using a similar light source, Lee et al. (2013) found that the human fingertip had epidermal and dermal extinction coefficient values of 1.64 and $0.95 \mathrm{~mm}^{-1}$, respectively. Thus, when comparing our experimental results with that of other studies, we were able to replicate the extinction coefficient of human skin.

The data obtained can be used to compute the amount of $\mathrm{TiO}_{2}$ required to produce a phantom with the desired extinction coefficient. This can be done by curve-fitting the averages

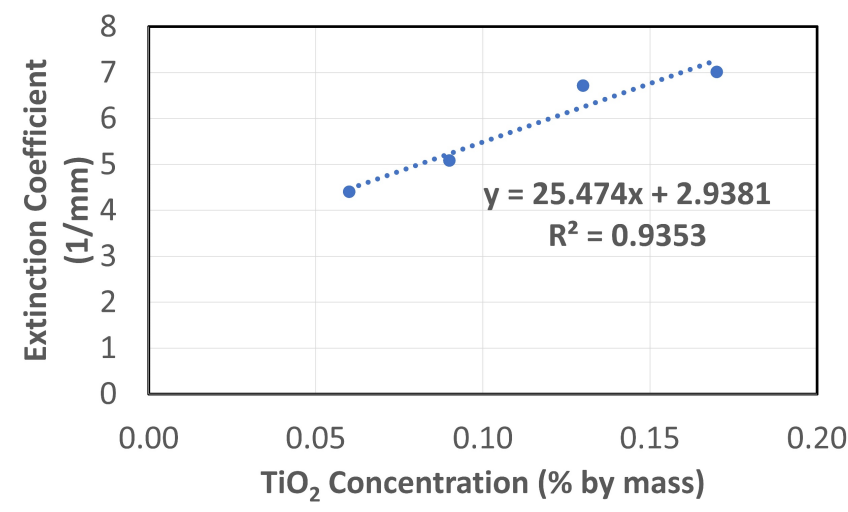

Figure 8. Curve-fitting to obtain an Equation Computing for the Extinction Coefficient

in Table 2 and determining the equation of the line shown in Figure 8 . The $0.02 \%$ data was excluded from the figure due to its high standard deviation. The derived equation is seen in Equation (5). The high R-squared value indicates that we can predict the extinction coefficient of the resulting phantom depending on the amount of $\mathrm{TiO}_{2}$ being added.

Required $\mathrm{TiO}_{2}$ conc.(\% by mass) $=\frac{\text { Desired Ext.coeff.-2.94 }}{25.47}$

For data verification through transmittance measurement, an inverse relationship between transmittance and $\mathrm{TiO}_{2}$ concentration was observed, shown in Figure 9. The percent transmittance shows a generally downward trend. This means that at lower concentrations of $\mathrm{TiO}_{2}$, more light passes through the sample, thus a higher transmittance. Conversely, the higher concentration of $\mathrm{TiO}_{2}$ makes the sample more turbid, and less light is transmitted. Even without $\mathrm{TiO}_{2}$, the maximum transmittance is just around $68 \%$ since gelatin by itself also dissipates light.

An interesting observation occurred for the transmittance for the phantom with $0.17 \% \mathrm{TiO}_{2}$ concentration. Light interacting with a particle is scattered in all directions. For our OCT probe with a numerical aperture of 0.14 , it has a narrow acceptance angle, only backscattered light at angles close to $180^{\circ}$ can 


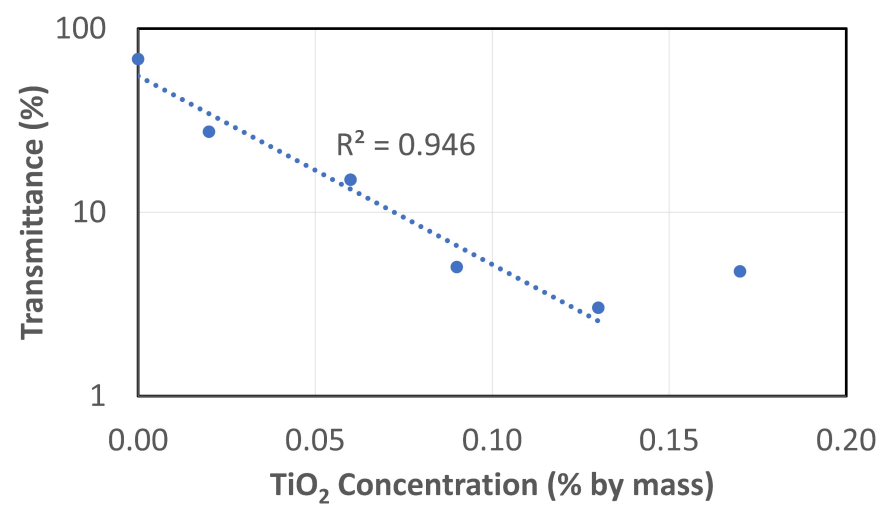

Figure 9. Plot showing the Inverse Relationship between Transmittance and $\mathrm{TiO}_{2}$ Concentration

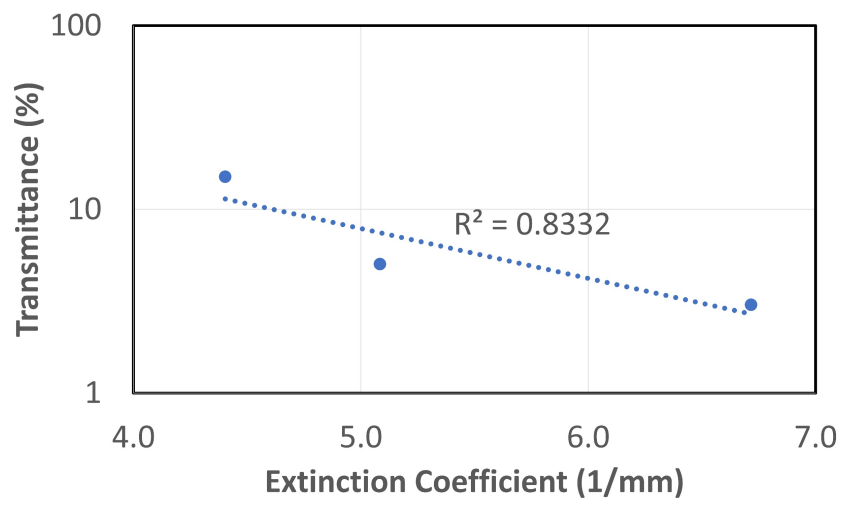

Figure 10. Plot showing the Inverse Relationship between Extinction Coefficient and Transmittance

reenter the probe. However, for the optical power meter, the sensor had a diameter of 1 centimeter hence a wide acceptance angle. The increased in concentration of scattering particles, increased the effect of multiple scattering which caused the attenuation change of transmittance for $0.17 \% \mathrm{TiO}_{2}$ phantom (Almasian et al., 2015; Kholodnykh et al., 2003; Peng and Shiina, 2017; Shiina, 2017).

By plotting percent transmittance on a logarithmic scale with extinction coefficient (which also exhibits logarithmic behavior based on the Beer-Lamber equation) in Figure 10, we can see a negative linear correlation between the two variables. A higher extinction coefficient means less light is transmitted across the phantom. In this correlation, the data for the $0.02 \%$ and $0.17 \% \mathrm{TiO}_{2}$ phantoms were excluded due to the errors brought about by the particle dispersion of the former, and the wide angle of acceptance of the optical power meter sensor of the latter as previously explained.

Another important information that can be extracted from A-scans is the depth of penetration of light. As seen in Figure 4 , the maximum penetration achieved in our experiment was around $0.4 \mathrm{~mm}$. Comparing this to commercial high-definition OCT (HD-OCT) models which can have a maximum skin

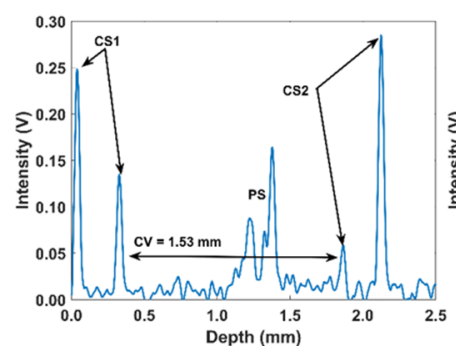

(a)

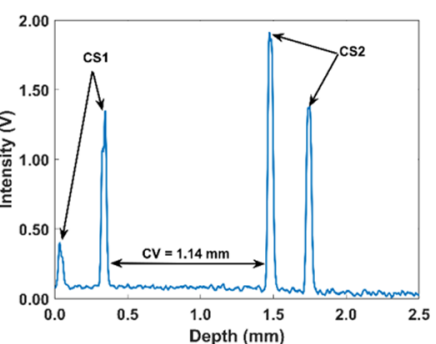

(b)
Figure 11. A-scan of an (a) Empty Sample Holder; (b) Filled with Gelatin Phantom: CS1, front Coverslip; CV, Cavity; CS2, Rear Coverslip; PS, Phantom Signal

penetration of around $0.57 \mathrm{~mm}$ Psomadakis et al., 2021, our fabricated phantoms and our own laboratory developed OCT system has good reliability.

\subsection{Group Refractive Index}

An A-scan of an empty phantom holder is shown in Figure 11(a). Each of the coverslips has two peaks representing the front and rear surfaces. The zero mark on the $\mathrm{x}$-axis represents the surface of the coverslip closest to the probe. The length of the empty cavity corresponds to the physical thickness of the cavity $(\mathrm{CV})$, in this case $\mathrm{d}=1.14 \mathrm{~mm}$. This same sample holder is filled with skin phantom, in this case, a $0.09 \%$ phantom with an A-scan shown in Figure 11(b). The thickness of the cavity increased to $1.53 \mathrm{~mm}$. This corresponds to the optical thickness $\Delta$. Then, by using Equation (4), the group refractive index can be computed.

Depending on the contents of the sample holder, the optical thickness of the cavity varies. The measured refractive indices with varying $\mathrm{TiO}_{2}$ concentrations are shown in Figure 12. It is observed that refractive index increases with increasing $\mathrm{TiO}_{2}$ concentration. The range of group refractive indices obtained were 1.32 to 1.48 . These values are close to the refractive index of water because the mass of the phantoms is mostly made up of water. More importantly, the experimental results are in close accordance with human skin refractive indices found in scientific literature, ranging from 1.34 to 1.56 (Khan et al., 2021 ).

Within the cavity, peaks due to the skin phantoms may appear such as the one in Figure 11 b, but they do not contribute to the computation of the refractive indices. Also, the front coverslip may not always have the tallest peak. This is because the distance of the probe to the sample holder should be adjusted so that the entire thickness of the sample holder can be detected. This is important especially for the phantoms with higher concentrations of $\mathrm{TiO}_{2}$. Consequently, the focal point is also shifted, hence the variations in intensities. Again, only the cavity thickness is necessary, and the signal intensities are not used in the computation of the group refractive index.

Using distilled water as the sample, the group refractive index we have obtained was 1.34 , which is close to 1.33 , the 


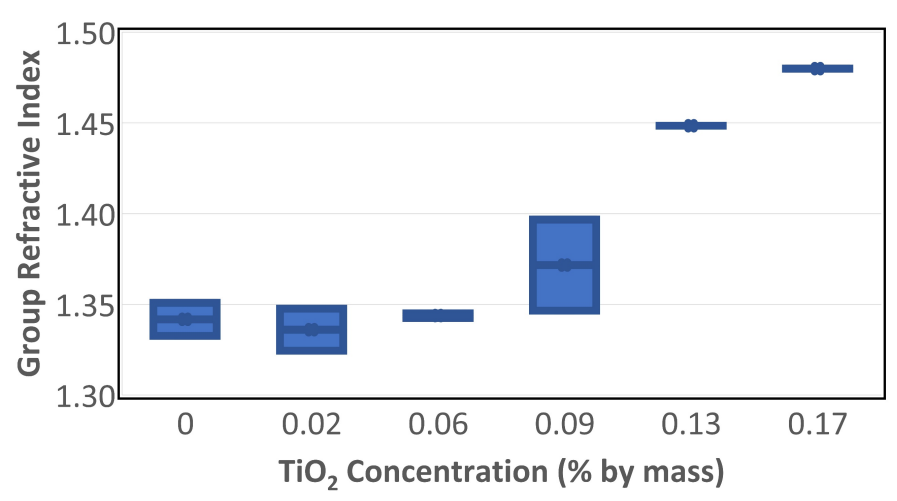

Figure 12. Relationship between Refractive Index and $\mathrm{TiO}_{2}$ Concentration

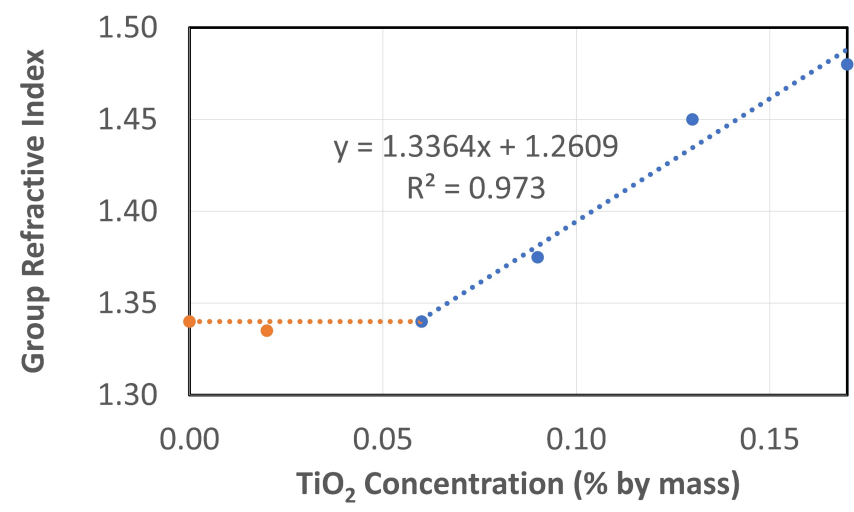

Figure 13. Curve-fitting to obtain an Equation Computing for the Refractive Index

textbook value for the absolute refractive index of water (Hecht, 2017). This means that the TD-OCT system can accurately measure the refractive index of water and possibly other aqueous solutions.

Figure 13 is the curve-fitting for the group refractive index for concentrations $0 \%$ to $0.17 \%$. The derived equation is shown in Equation (6). The line starting from zero just indicates that at $\mathrm{TiO}_{2}$ concentrations lower than $0.02 \%$, the refractive index can be assumed to be relatively constant at 1.34 , our experimental value for the refractive index of water. Since much of the mass of the phantom is due to water, it follows that the refractive index is largely attributed to the water content. By forming a curve fit starting from $0.02 \%$ to $0.17 \%$, we can see a linear relationship between group refractive index and $\mathrm{TiO}_{2}$ concentration. Hence, we can easily control the group refractive index of the phantom just by varying the $\mathrm{TiO}_{2}$ concentration.

$$
\text { Required } \left.\mathrm{TiO}_{2} \text { conc.(\% by mass }\right)=\frac{\text { Desired Ref.Ind. }-1.26}{1.34}
$$

\section{CONCLUSIONS}

We were able to determine the extinction coefficients and refractive indices of the gelatin-based skin phantoms. In the study, both the extinction coefficient and group refractive index had positive correlations with $\mathrm{TiO}_{2}$ concentration. The increase in extinction coefficient due to increased $\mathrm{TiO}_{2}$ concentration was verified by a decrease in transmittance, in other words, a negative correlation. Our results for the extinction coefficient and group refractive index agree with existing literatures. Extinction coefficient increases with $\mathrm{TiO}_{2}$ concentration (Vermeer et al., 2014), we were able to fabricate skin phantoms that replicate the extinction coefficient of human skin (Adili and Shiina, 2018; Lee et al., 2013). For refractive index studies, our measurement is close to the standard value for water (Hecht, 2017). This is significant since water forms the bulk of most human tissue, whose refractive indices our phantoms also successfully replicated (Khan et al., 2021).

Therefore, we were able to show that it is possible to fabricate skin phantoms that mimic human skin. By varying the concentration of $\mathrm{TiO}_{2}$, we can simulate skin from different anatomical sites, or different skin properties depending on age, sex, and even various skin conditions. This is useful for studying skin diseases and improving the capability of TD-OCT as a diagnostic tool for the human skin. This research can then be expanded to include other tissue types, as there are still more possible applications of OCT outside ophthalmology and dermatology.

\section{ACKNOWLEDGEMENT}

The authors acknowledge the Commission on Higher Education (CHED) of the Philippine Government for funding the project entitled "Development of a Portable Optical Coherence Tomography System for the Evaluation of Human Skin Analogues". The authors also acknowledge DLSU URCO with project number $47 \mathrm{~F}$ U 2TAY18-2TAY19 and CENSERDLSU for supporting the project.

\section{REFERENCES}

Adili, D. and T. Shiina (2018). A quantitative evaluation of skin phantom by skin TD-OCT. Proceedings of The $19^{\text {th }}$ Coherent Laser Radar Conference; 19-22

Akarçay, H. G., S. Preisser, M. Frenz, and J. Rička (2012). Determining the optical properties of a gelatin- $\mathrm{TiO}_{2}$ phantom at $780 \mathrm{~nm}$. Biomedical Optics Express, 3(3); 418-434

Almasian, M., N. Bosschaart, T. G. van Leeuwen, and D. J. Faber (2015). Validation of quantitative attenuation and backscattering coefficient measurements by optical coherence tomography in the concentration-dependent and multiple scattering regime. Journal of Biomedical Optics, 20(12); 121314

Chang, S. and A. K. Bowden (2019). Review of methods and applications of attenuation coefficient measurements with optical coherence tomography. Journal of Biomedical Optics, 24(9); 90901 
Cheong, W., S. Prahl, and A. Welch (1990). A review of the optical properties of biological tissue. IEEE Journal of Quantum Electronics, 26(12); 2166-2185

Cook, J. R., R. R. Bouchard, and S. Y. Emelianov (2011). Tissue-mimicking phantoms for photoacoustic and ultrasonic imaging. Biomedical Optics Express, 2(11); 3193-3206

Faber, D. J., F. J. Van Der Meer, M. C. Aalders, and T. G. van Leeuwen (2004). Quantitative measurement of attenuation coefficients of weakly scattering media using optical coherence tomography. Optics Express, 12(19); 4353-4365

Forouzeshfard, M. R., S. Ghafari, and Z. Vafapour (2021). Solute concentration sensing in two aqueous solution using an optical metamaterial sensor. Journal of Luminescence, 230; 117734

Fujimoto, J., D. Huang, E. Swanson, C. Lin, J. Schuman, W. Stinson, W. Chang, M. Hee, T. Flotte, K. Gregory, and C. Puliafito (1991). Optical Coherence Tomography. Science

Fujimoto, J. G. and W. Drexler (2015). Optical Coherence Tomography: Technology and Applications. Springer

Gambichler, T., R. Matip, G. Moussa, P. Altmeyer, and K. Hoffmann (2006). In vivo data of epidermal thickness evaluated by optical coherence tomography: effects of age, gender, skin type, and anatomic site. Journal of Dermatological Science, 44(3); 145-152

GMIA (2019). Gelatin Handbook. GELITA North America

Hecht, E. (2017). Optics. Pearson Education Limited

Huang, R., Q. Zhang, P. Qi, and W. Liu (2019). Concentration Measurement of Uniform Particles Based on Backscatter Sensing of Optical Fibers. Water, 11(9); 1955

Izatt, J. A., M. A. Choma, and D. A (2015). Theory of Optical Coherence Tomography. Optical Coherence Tomography: Technology and Applications

James, W., D. Elston, J. Treat, M. Rosenbach, and I. Neuhaus (2020). Andrewes' Diseases of the Skin - Clinical Dermatology. Elsevier

Khan, R., B. Gul, S. Khan, H. Nisar, and I. Ahmad (2021). Refractive index of biological tissues: Review, measurement techniques, and applications. Photodiagnosis and Photodynamic Therapy, 33; 1-9

Kholodnykh, A. I., I. Y. Petrova, M. Motamedi, and R. O. Esenaliev (2003). Accurate measurement of total attenuation coefficient of thin tissue with optical coherence tomography. IEEE Journal of Selected Topics in Quantum Electronics, 9(2); 210-221

Kim, Y. H. (2016). Ultrasound phantoms to protect patients from novices. The Korean Journal of Pain, 29(2); 73

Lee, C.-K., M.-T. Tsai, F.-Y. Chang, C.-H. Yang, S.-C. Shen, O. Yuan, and C.-H. Yang (2013). Evaluation of moisturerelated attenuation coefficient and water diffusion velocity in human skin using optical coherence tomography. Sensors, 13(4); 4041-4050

Liu, P. (2014). Optical Coherence Tomography for Material Characterization. Thesis. Nanjing University of Aeronautics and
Astronautics

Marques, M. J., R. Green, R. King, S. Clement, P. Hallett, and A. Podoleanu (2021). Sub-surface characterisation of latestgeneration identification documents using optical coherence tomography. Science and Justice, 61(2); 119-129

Maruyama, H., S. Inoue, T. Mitsuyama, M. Ohmi, and M. Haruna (2002). Low-coherence interferometer system for the simultaneous measurement of refractive index and thickness. Applied Optics, 41(7); 1315-1322

Pant, C., M. S. Olyaee, and A. Rastogi (2017). Advanced imaging and therapeutic endoscopy. Techniques in Gastrointestinal Endoscopy, 19(3); 151-158

Peng, Z. and T. Shiina (2017). Generation control of a nondiffractive beam in random media by adjusting concentration. Optics Communications, 391; 94-99

Psomadakis, C. E., N. Marghoob, B. Bleicher, and O. Markowitz (2021). Optical Coherence Tomography. Clinics in Dermatology

Rateria, A., M. Mohan, K. Mukhopadhyay, and R. Poddar (2019). Investigation of Puccinia triticina contagion on wheat leaves using swept source optical coherence tomography. Optik, 178; 932-937

Schneider, S. L., I. Kohli, I. H. Hamzavi, M. L. Council, A. M. Rossi, and D. M. Ozog (2019). Emerging imaging technologies in dermatology: Part I: Basic principles. Journal of the American Academy of Dermatology, 80(4); 1114-1120

Shiina, T. (2017). Non-diffractive beam in random media. Optics Communications, 398; 12-17

Shiina, T., Y. Moritani, M. Ito, and Y. Okamura (2003). Longoptical-path scanning mechanism for optical coherence tomography. Applied Optics, 42(19); 3795-3799

Tuchin, V. V., A. Bashkatov, E. Genina, V. Kochubey, V. Lychagov, S. Portnov, N. Trunina, D. Miller, S. Cho, H. Oh, et al. (2011). Finger Tissue Model and Blood Perfused Skin Tissue Phantom. Dynamics and Fluctuations in Biomedical Photonics VIII. International Society for Optics and Photonics

Ugryumova, N., S. J. Matcher, and D. P. Attenburrow (2004). Measurement of bone mineral density via light scattering. Physics in Medicine and Biology, 49(3); 469-483

Vermeer, K. A., J. Mo, J. J. Weda, H. G. Lemij, and J. F. de Boer (2014). Depth-resolved model-based reconstruction of attenuation coefficients in optical coherence tomography. Biomedical Optics Express, 5(1); 322-337

Yang, H., G. Zheng, and M.-c. Li (2008). A discussion of noise in dynamic light scattering for particle sizing. Particle and Particle Systems Characterization, 25(5); 406-413

Yang, Z., J. Shang, C. Liu, J. Zhang, and Y. Liang (2020). Identification of oral cancer in OCT images based on an optical attenuation model. Lasers in Medical Science, 35(9); 1999-2007

Yeh, Y.-L. (2008). Real-time measurement of glucose concentration and average refractive index using a laser interferometer. Optics and Lasers in Engineering, 46(9); 666-670 